Uwe Berlit

\title{
Das Grundgesetz und die »Fremden« Bürger, Volk und Nation bei der Entstehung des Grundgesetzes ${ }^{1}$
}

\section{Einleitung}

Die Unterscheidung zwischen dem »Bürger « und dem »Fremden « dient traditionell auch der Bestimmung der im Staat verkörperten Nation. ${ }^{2}$ Die mit der staatlichen Einigung Deutschlands und dem Prozeß der europäischen Integration verbundene Renaissance des Konzepts der "Nation « ${ }^{3}$ lenkt den Blick auf den Umgang mit dieser Frage bei der Entstehung des Grundgesetzes. Ihre Darstellung soll zur Klärung beitragen, ob die gegenwärtige »Rück «besinnung auf Nation oder Nationalstaatlichkeit konzeptionell anknüpfen kann an Überlegungen, die bereits bei der Schaffung des Grundgesetzes selbst angelegt waren, oder ob sie unter Berufung auf tatsächliche Probleme auf einen Rückschritt gegenüber der Weitsicht der Verfassungsschöpfer hinausläuft.

Die Beratungen über das Grundgesetz fielen in eine Zeit, in der der Begriff der Nation ${ }^{4}$ durch den Nationalsozialismus nachhaltig belastet war, ohne daß das europäische Konzept der Nationalstaatlichkeit's bereits überwunden war. Wegen der Teilung des Deutschen Reiches als dem bisherigen staatlichen Ausdruck der »deutschen Nation ${ }^{6}{ }$ und dem Festhalten am Ziel der nationalen Einheit konnte und sollte es nicht die Verfassung einer Nation sein. Das klassische Problem der Grenzziehung nach außen, die eine Nation ausmacht, wurde überlagert durch das Problem der "deutschen Einheit«, an der in dieser Phase beide Seiten festgehalten haben, die territorialen Verschiebungen im Osten des früheren Deutschen Reiches und die Folgen eines rassisch-völkisch aufgeladenen Nationenbegriffs, der das schon bei der Reichsgründung $1870 / \mathrm{I}^{7}$ fragile Kriterium »ethnischer « Homogenität durch gewaltförmige Ausgrenzungen bis hin zu massenhafter physischer Vernichtung noch fragwürdiger hat werden lassen.

Tatsächlich trafen die Grundgesetzgeber als Folge der Mordtaten der Nazis und der kriegsbedingten Gebietsveränderungen auf ein zwangsweise homogenisiertes Volk, das für ethnische Ab-oder Ausgrenzungen deutscher Staatsangehöriger - sei es nach Selbstverständnis oder durch Zuschreibung - keine Anknüpfungspunkte mehr bot.

I Überarbeiteter Beitrag zum 5. Chiemsee-Verfassungsgespräch „Der Burger und der Fremde $₫$ im Sommer 1993

2 Insoweit konvergieren die Begriffe "Volk« und "Nation«, die letztere auf die "Staatsnation « reduziert Zum Begriff der Kulturnation s. Heike Puppe, Die kulturelle Identitat der Deutschen, Munster 1990, $145 \mathrm{ff}$.

3 Zur »Themenkonjunktur« des Nationalstaatskonzepts s. Karlheinz Weißmann, Wıederkehr eines Totgesagten: Der Nationalstaat am Ende des 20. Jahrhunderts, APuZ B 14/93, $3 \mathrm{ff}$.

4 Nation hier verstanden als »Ideal einer Solidargemennschaft gleichberechtigter, freier Burger « (Rolf Grawert, Staatsvolk und Staatsangehorigkeit, in: HdBStR Bd. I, Heidelberg $1987, \$ 14$ Rdn. 10).

s S. dazu M. Rainer Lepsius, Der eu ropaische Nationalstaat: Erbe und Zukunft, in: ders., Interessen, Ideen und Institutionen, Opladen 1990, $256 \mathrm{ff}$; zu den Faktoren der Konstruktion der Differenz zwischen Eigen- und Fremdgruppe im Bewußtsein der Bevolkerung vgl. D. Fuchs/J. Gerhards/E. Roller, Wir und die anderen. Ethnozentrismus in den zwolf Ländern der europäischen Gemeinschaft, KZfSS 1993, $238 \mathrm{ff}$.

6 Zur T'hese von der deutschen Nation, die ihren Natıonalstaat im Deutschen Reich hatte, als Reichsnation s. Otto Dann, Nation und Nationalismus in Deutschland 1770-1990, München 1993, 16 ff., $310 \mathrm{ff}$.

7 Die Reichsgrundung kann nicht als Akt eines (bereits geformten, präexistenten) Staatsvolkes, sondern als staatsvolkbildender Akt gewertet werden (Grawert (Fn. 4$\rangle, \$$ i $_{4}$ Rdn. I3). 
Die Flüchtlinge galten bei allen sozio-ökonomischen und kulturellen Unterschie$\operatorname{den}^{8}$ und damit auch "Fremdheit" unbestritten nicht nur als deutsche Staatsangehörige, sondern als ihrer Herkunft nach und kulturell Deutsche. ${ }^{9}$ Auf der Ebene des Gesamtstaats waren verfassungsgesetzlich Fragen des Umgangs mit nationalen oder ethnischen Minderheiten nicht zu bewältigen; Minderheiten wurden allein als innerstaatliche, konfessionelle oder landsmannschaftliche bei der Konzeption der Schulartikel wahrgenommen, nicht als nationale angesehen. ${ }^{10}$ Die mit den Fü̈chtlingen und Vertriebenen verbundenen Zuwanderungs- und Integrationsprobleme überlagerten zudem quantitativ jedes seinerzeit vorstellbare Ausländerintegrationsproblem: 1946 lebten in den Westzonen 5,7 Mio Vertriebene ( $13,1 \%$ der Bevölkerung), bis 1950 wuchs ihre Zahl auf 7,88 $\mathrm{Mio}^{11}$ (16,5\%).

Obwohl die Verfassunggebung der Westdeutschen ein von den alliierten Besatzungsmächten nach Grund und Richtung mitinitiierter Prozeß war und in diesem Sinne nicht freie Assoziation von Bürgern ${ }^{12}$, erstreckten sich deren Vorgaben, begleitende Kontrolle und Interventionen thematisch nicht auf die Normen mit Bezug zum "Fremden«. Umgekehrt klingt das in der Bevölkerung verbreitete Gefühl von der Besatzungsherrschaft als $\gg$ Fremdherrschaft $\alpha^{13}$ in einer vom sog. Fünferausschuß vorgeschlagenen Fassung der Präambel ${ }^{14}$ an, die darauf hinweist, die Verfassunggebung sei auch in der Erkenntnis erfolgt, »daß die Besetzung Deutschlands durch fremde Mächte die Ausübung eines freien nationalen Selbstbestimmungsrechts schweren Einschränkungen unterworfen hat«.

Entstehungsgeschichtlich macht sich das Problem der nationalen Grenzziehung fest u. a. an der Bestimmung des Begriffs des "Deutschen « (Art. × 6 GG), des Umgangs mit politisch Verfolgten (Art. 16 GG), den Rechten und Pflichten, die den »Fremden « im politischen, wirtschaftlichen und sozialen Bereich eingeräumt bzw. auferlegt werden, an der Unterscheidung von Bürger- und Menschenrechten, und - last not least - an der staatsbürgerlichen Gleichheit. ${ }^{\text {'s }}$ Der Blick auf die Behandlung dieser Fragen bei der Gestaltung des Grundgesetzes deutet darauf, daß in das Grundgesetz als Zukunftsentwurf Prinzipien eingeflossen sind, die weitsichtigere als die heute vorherrschenden Antworten auf die aktuell drängenden Fragen nahelegen, wie sich das Gemeinwesen selbst definiert und gegen das Fremde abgrenzt, in welcher Weise und in welchem Umfang dem fremden Dritten (ideelle oder materielle) Solidarität geschuldet ist.

$8 \mathrm{Vgl}$. P. Waldmann, Die Eingliederung der ostdeutschen Vertriebenen in die westdeutsche Gesellschaft, in: J. Becker/T. Stammen/P. Waldmann (Hrsg.), Vorgeschichte der Bundesrepublik Deutschland, 2. Aufl., Munchen $1987,165 \mathrm{ff}$.

9 Dazu M. Lepsius, „Ethnos« oder »Demos «, in: ders.: Interessen, Ideen und Institutionen, Opladen 1990, 247.

10 Dies ist einer der Hintergrunde fur das Recht der Eltern, uber die Teilnahme ihrer Kinder am Religionsunterricht zu bestimmen (Art. 7 Abs. 2 GG), und die Privatschulfreiheit (Art. 7 Abs. 4 GG) (dazu JöR N.F. Bd. I, Ior ff.).

11 Ohne die ca. 1,5 Mio aus der SBZ und Berlin (Ost) Zugewanderten; Angaben bei Waldmann (Fn. 8), 169.

12 Besonders diesen Aspekt wollte der Abg. von Mangoldt unter Berufung auf das Selbstbestimmungsrecht in der Praambel verankert sehen (Grundsatzausschuß, 8. Sitz. v. 7. 10. 1948, KurzProt S. 2) (ferner: Teilordnungs-, Kontinuitäts- und Provisoriumsgedanke); ähnlich der Abg. Schmid, der für die Aufnahme des Begriffs der "Fremdherrschaft " plädierte.

13 Josef Foschepoth, Zur deutschen Reaktion auf Niederlage und Besatzung, in: L. Herbst (Hrsg.), Westdeutschland 1945-1955, Munchen 1986, 151 (156 ff.). Zur Verknupfung dieser Vorbehalte mit dem demokratischen Gedanken im Denken Schumachers vgl. Carlo Schmid, Erinnerungen, Bern/Munchen/Wien, s. Aufl. 1979, 318.

14 S. Hauptausschuß, 47. Sitz., 8. 2. 1949, KurzProt 5.

is Fragen der Selbstverortung Deutschlands in der Welt und insb. seıner Öffnung hin zu Europa (Art. 24 GG) mit der spezifischen und bewußten Modifikation des klassischen Konzepts des westeuropaischen Nationalstaats soll hier nicht weiter nachgegangen werden. 
Der Verfassungsentwurf von Herrenchiemsee setzt in seiner Präambel das deutsche Volk ${ }^{16}$ als verfassunggebende Kraft voraus. Volks- oder Staatszugehörigkeit werden nicht näher definiert. Deutlicher als die Präambel des Grundgesetzes, die den Willen zur Wahrung nationaler und staatlicher Einheit ${ }^{17}$ nur als Motiv der Schaffung des Grundgesetzes als Übergangsordnung hervorhebt, beruft sich der Mehrheitsvorschlag in Herrenchiemsee noch auf das unverzichtbare Recht des Volkes auf Gestaltung seines nationalen Lebens.

Der Parlamentarische Rat mußte sich bei der Diskussion des heutigen Art. I 6 GG aus zwei Gründen intensiver mit Fragen der Deutscheneigenschaft und der Staatsangehörigkeit beschäftigen: Zum einen setzte sich im Parlamentarischen Rat bei den Grundrechten eine Unterscheidung von Menschen- und Bürgerrechten durch, während der Herrenchiemseer Entwurf - mit Ausnahme des Wahlrechts - durchgängig universalistisch konzipierte Menschenrechte garantieren wollte. Dies machte eine genauere Umschreibung der Grundrechtsträger unumgänglich.

Dies hat frühzeitig der Allgemeine Redaktionsausschuß klar hervorgehoben: »Nach Ansicht des Ausschusses ist eine Definition der vorgeschlagenen Art in den Übergangsbestimmungen unumgänglich notwendig, ohne daß deshalb einer $-\mathrm{z}$. B. aus politischen Gründen - erforderlich werdenden Neuregelung durch einfaches Gesetz vorgegriffen werden muß. Der Vorschlag des Ausschusses macht den besonderen Begriff eines `Bundesangehörigen<, der mit Rücksicht auf die Ostzone vermieden werden sollte, überflüssig; zugleich werden außer den deutschen Reichsangehörigen auch sämtliche volksdeutschen Flüchtlinge, gleichgültig in welcher Besatzungszone sie leben, sowie die Angehörigen nichtdeutschen Volkstums, die mit volksdeutschen Flüchtlingen verheiratet sind oder von ihnen abstammen und wegen dieser Familienzugehörigkeit aus ihrem Heimatstaat abgewiesen worden sind, miterfaßt « ${ }^{18}$

Zum anderen galt es vor dem Hintergrund von Umsiedlungen, Vertreibungen, Gebietsgewinnen und -verlusten während und nach dem Krieg einen Staatsangehörigkeitsbegriff zu entwickeln, der - »mit Rücksicht auf die Ostzone « $^{19}$ - auf den als zu eng empfundenen Begriff des Bundesangehörigen verzichtete und auf die unklar gewordenen Grenzen des Staatsgebiets reagierte.

Die Anknüpfung an die "alte« Reichsstaatsangehörigkeit und die Grenzen des Reiches von 1937 unterstrich die im Parlamentarischen Rat mehrheitlich vertretene Kontinuitätsthese; damit wurde zugleich eine Stellungnahme zu den von den Nationalsozialisten verfügten Ausbürgerungen unausweichlich.

Art. I 6 GG konzipiert die Bundesrepublik formell als Staatsbürgergesellschaft: Jeder Staatsangehörige ist auch Deutscher. Die durch Staatsbürgerschaft vermittelte Zugehörigkeit zum »Demos $\aleph^{20}$ hängt nicht notwendig davon ab, auch Teil des »Eth-

16 Der Streit im Konvent von Herrenchiemsee, ob »das Volk oder die Lander die verfassunggebende Gewalt innehaben, hängt eng mit der Diskontinuttatsthese zusammen, nach der mit dem Deutschen Reich auch das deutsche Volk als Nation untergegangen sei (dazu Berıcht uber den Verfassungskonvent auf Herrenchiemsee, Munchen 1948, 18f.). Die Präambel indiziert die Durchsetzung des Fortbestandsdogmas (Grawert $\left\langle\right.$ Fn. 4 ), $\$_{14}$ Rdn. I6)

17 Hierin zeigt sich ein eher organologischer Volksbegriff (Grawert $\langle$ Fn. 4$\rangle, \bigotimes 14$ Rdn. 6)

18 Anm. 28 zu der vom Allgemeinen Redaktionsausschuß in der ersten Lesung formulierten Fassung der Art. 2 I bis 44 (Stand: 16. II. 1948) (PR I1.48-279/II); der Hinweis auf die Ausweisung deutet verfassungsgeschichtlich auf einen engen Flüchtlingsbegriff, der unmittelbaren Vertreibungsdruck voraussetzt.

19 Begründung zum Vorschlag des Allgemeinen Redaktionsausschusses eines Art. I38b vom I 8. 12. 1948, PR Drucks. 29 I.

20 Hier verstanden als Staatsvolk, also als die Gesamtheit der Staatsangehörigen (also unter Ausschluß der sog. Statusdeutschen). 
nos« zu sein ${ }^{2 ז}$. Die über die Deutscheneigenschaft vermittelte Staatsangehörigkeit erlaubt, für die »Identität« der Gemeinschaft auf Kriterien ethnisch-kultureller Gemeinsamkeiten zu verzichten und statt dessen auf die gemeinsame Praxis von Bürgerinnen und Bürgern abzustellen, die ihre demokratische Teilnahme- und Kommunikationsrechte aktiv wahrnehmen.

Dieser enge Zusammenhang von Staatsangehörigkeit und politischen Rechten kommt in der - besorgten - Frage des Abg. von Mangoldt zum Ausdruck ${ }^{22}$, daß hiermit auch »den Bewohnern der östlichen Gebiete das Wahlrecht in den Ländern des Westens « gegeben werde. ${ }^{23}$ Mit der Ausdehnung auf die Flüchtlinge und Vertriebenen war aber klar, daß der Schutz des zu schaffenden Solidarverbandes ${ }^{24}$ auch auf Personen ohne deutsche Staatsangehörigkeit erstreckt werden sollte.

Das allgemeine Spannungsverhältnis zwischen der durch Geburt - nach welchem Prinzip auch immer - vermittelten Deutscheneigenschaft und der gedanklich hiervon zu trennenden Staatsangehörigkeit wurde dem Parlamentarischen Rat zum Themawenn auch nur unter dem eingeschränkten Blickwinkel des Wiedereinbürgerungsanspruchs $^{25}$ der von den Nationalsozialisten ausgebürgerten Personen. ${ }^{26}$ Die Frage war, ob die Ausbürgerungen der Nazis als gültig anzuerkennen waren ${ }^{27}$ mit der Folge, daß es eines konstitutiven Aktes bedurfte, um die deutsche Staatsangehörigkeit wieder zu erlangen, ob alle Ausbürgerungen ex tunc auch dann aufgehoben werden sollten, wenn die Ausgebürgerten inzwischen eine andere (politische) Heimat gefunden hatten, oder ob die deutsche Staatsbürgerschaft automatisch zumindest all jenen zuerkannt werden solle, die nach Deutschland zurückgekehrt waren.

Die vom Prinzip bestmöglicher Wahrnehmung der ideellen und finanziellen Belange der Ausgebürgerten geprägte Debatte anerkennt, daß Staatsangehörigkeit immer auch ein voluntatives Element birgt: Kein Ausgebürgerter sollte gegen seinen Willen wieder zum Deutschen gemacht werden. Aus historisch-moralischen Gründen sollte die deutsche Staatsangehörigkeit aber auch nicht von unzumutbaren Erschwerungen abhängen. Diese Rücksichtnahme führte im Zuge der vielfältigen Formulierungsänderungen dazu, daß parteiübergreifend auf die zeitweilige betonte Wiedereingliede-

$21 \mathrm{Zu}$ dieser Unterscheidung vgl. Lepsius (Fn. 9), 249. J. Habermas (Staatsbirgerschaft und nationale Identität, in: ders.: Faktizitat und Geltung, Frankfurt 1 $99^{2}, 6{ }_{32}$ ff. $\left.\langle 633,636\rangle\right)$ greift dies mit der Unterscheidung von der Nation als einer vorpolitischen Einheit einer historischen Schicksalsgemeinschaft und der einer notwendig universalistisch gegrundeten politischen Staatsburgernation auf.

22 Die Nationalsozialisten hatten diesen Konnex durch die Unterscheidung der (passiven) Staatsangehörigkeit und der (aktiven) Reichsburgerschaft aufgehoben (vgl. Grawert $\langle$ Fn. 4$\rangle, \$_{14}$ Rdn. 34).

23 Hauptausschuß, s. Sitz., 18.11. 1948, Prot.64; der Abg. Dehler verwes die Frage dann an das einfachgesetzliche Wahlrecht; in der 26. Sitzung des Grundsatzausschusses vom 30.11.1948 ubernahm der Grundsatzausschuß den Vorschlag des Abg. v. Mangoldt auf Erganzung des Art. 14 I: "Wer wahlberechtigt ist, entscheiden Verfassung oder Gesetz«.

24 Diese Schutzdimension wollte die DP mit ihrem Antrag vom 19. 11. 1948, ein Menschenrecht auf Heimat zu schaffen, betonen (PR 11.48-298 v. 19.11.1948: "Jeder Mensch hat das Recht auf eine Heimat. Die vornehmstc Pflicht des Staates ist, seinen Bewohnern Heimat, Geborgenheit und Frieden zu gewährleisten $\alpha$ ).

25 Erstmals entwickelte der Grundsatzausschuß hierzu einen Formulierungsvorschlag, und zwar in der 30. Sitz. v. 6. 12. 1948 :

Art. 1 $38 \mathrm{bb}$ : Fruhere deutsche Staatsangehorige, denen in der Zeit zwischen dem 30. Januar 1933 und 8. Mai 1945 die Staatsangehorigkeit aus politıschen, rassischen oder religiosen Grunden entzogen worden ist, und ihre Abkömmlinge sind auf thren Antrag wieder einzuburgern " (KurzProt 2 〈PR 12.48-360 II $\rangle$ ).

26 Auf dieses Problem machte in der 18. Sitzung des Hauptausschusses der Abg. Lowenthal aufmerksam (4. 1 2. 1948, KurzProt 5; PR 1.49-456; Prot. 217). Der Abg. Schmid verwies darauf, daß sich der Kontrollrat das Recht vorbehalten habe, sdie Staatsangehorigkeit zu verleihen und wiederzuverlerhen" (ebd., 217). In Fragen der Wiederverleihung der Staatsangehorigkeit an Ausgebürgerte scheint es eine nach Besatzungszonen divergierende Praxis gegeben zu haben (s.a. v. Mangoldt, ebd. 217)

27 Diesen Aspekt hebt der selbst ausgebürgerte Abg. Renner (KPD) immer wieder hervor (etwa Prot. S. 677) 
rungsvoraussetzung "Staatenlosigkeit oder Aufgabe einer anderen Staatsangehörigkeit ${ }^{28}$ verzichtet $^{29}$ wurde. Gegen eine Tendenz im internationalen Recht wurde damit bewußt die Möglichkeit einer doppelten Staatsangehörigkeit hingenommen. Gefahren durch elementare Loyalitätskonflikte, die heute vielfach gegen Doppelstaatsangehörigkeit ins Feld geführt werden, wurden dabei nicht als durchgreifend gesehen. Das Problem wurde aber auch nicht übersehen: Der Abg. Schmid ${ }^{30}$ sprach sich für die Möglichkeit doppelter Staatsangehörigkeit nicht zuletzt deswegen aus, weil dies ein Mittel zur Relativierung nationalstaatlichen Denkens sei.

Der das deutsche Staatsangehörigkeitsrecht zumindest seit 1913 prägende Abstammungsgrundsatz (jus sanguinis) war dem Parlamentarischen Rat weder Problem noch Anliegen. Das Prinzip, das die Staatsangehörigkeit vermittelt, ist nicht erörtert, eine Festlegung auf einen der völkerrechtlich anerkannten und möglichen Anknüpfungspunkte für die Aufnahme in die deutsche Staatsangehörigkeit nicht getroffen worden. Die Gestaltungsfreiheit des einfachen Bundesgesetzgebers ist mithin auch heute nicht begrenzt. Mangels klarer Entscheidung der Verfassung erlaubt die Fortgeltung des bei Inkrafttretens geltenden Staatsangehörigkeitsrechts insbesondere nicht den Umkehrschluß, der Übergang zum jus soli - als einzigem oder ergänzendem Prinzip - sei einfachgesetzlich nicht möglich oder verletze die Verfassungs»identität«. Aus der Entstehungsgeschichte ergeben sich jedenfalls keine Hinweise darauf, daß das Abstammungsprinzip ausnahmslos gegenüber dem Territorialitätsprinzip festgeschrieben werden sollte und allenfalls die Einbürgerung Abstammungsgrenzen überwinden helfen sollte.

Durch die Erstreckung der besonderen Stellung des "Statusdeutschen« auf die Abkömmlinge wird das Abstammungsprinzip allerdings implizit betont. Im Gegensatz zu der durch die formelle Staatsangehörigkeit vermittelten, von ethnischen Aspekten äußerlich freien "Deutscheneigenschaft " schwingt in der Sache im Begriff der deutschen Volkszugehörigkeit der Gedanke der durch Geburt vermittelten Abstammungs- und Schicksalsgemeinschaft mit. ${ }^{31}$ Der besondere Schutz geflüchteter oder vertriebener Volksdeutscher war dem Parlamentarischen Rat indes derart selbstverständlich, daß sich der Entstehungsgeschichte weder Anhaltspunkte für seine Ausfüllung noch für den umfassenden Gesetzesvorbehalt entnehmen lassen, der seinem Wortlaut nach auch erlaubt, den Flüchtlingen und Vertriebenen die Deutscheneigenschaft und damit einen Einbürgerungsanspruch zu verwehren. Die von der Gesetzgebung im Vertriebenenrecht alter Fassung sehr weit gezogenen Grenzen deutscher Volkszugehörigkeit sind jedenfalls vom Verfassunggeber so nicht vorgezeichnet.

\section{Ausbürgerung, Auslieferung und Asyl}

Eine klare Trennlinie zwischen den besonders geschützten Staatsangehörigen und den Fremden, zwischen Innen und Außen, zeigt Art. I6 GG: Die Asylrechtsgewährung in der Fassung von 1949 galt als Ausdruck besonderer Fremdenfreundlichkeit des Grundgesetzes, und er vermittelte mit dem Ausbürgerungs- und Auslieferungsverbot auch Deutschen spezifischen Schutz.

28 Vorschlag des Allgemeinen Redaktionsausschusses.

29 Hauptausschuß, 39. Sitzung, Kurzprot., S. 4 f. $(485$ f.)

30 Hauptausschuß, 39. Sitzung, 486.

31 In dem einfachgesetzlıchen Bekenntniserfordernıs wird auf die Selbstzuordnung zur Kulturgemeinschaft abgestellt, die durch objektive "Kulturfaktoren " bekraftigt werden muß (vgl. Grawert $\langle$ Fn. 4 $\rangle$, Rdn. 30). Die hohe Bedeutung, die von unterschiedlichsten Motiven und Faktoren getragene Eintragungen in Volkslisten bzw. die Wehrdienstlesstung fur die Volkszugehorigkeitsprufung erlangt haben, deutet allerdings auf außerst unscharfe Grenzen des "Kultur«begriffs, der sich vom klassischen Modell der „Kulturnation « abhebt. 
Das Menschenrecht auf eine durch Staatsangehörigkeit dokumentierte "politische Heimat « unterstreicht bereits das Verbot des Entzuges der deutschen Staatsangehörigkeit. ${ }^{32}$ Es ist im Zuge der Beratungen sachlich und personell erweitert worden: Zunächst ging es nur um den willkürlichen bzw. auf politische Motive gestützten Entzug der Bundeszugehörigkeit, der zeitweilig noch durch eine an die Weimarer Reichsverfassung anknüpfende Regelung ergänzt war, die die Bundeszugehörigkeit an die Landesangehörigkeit band. Motiv für das strikte, ausnahmslose Entzugsverbot war vor allem die Ausbürgerungspraxis der Nationalsozialisten; daneben wurde auch hier für eine Überwindung nationalstaatlichen Denkens und eine Abkehr vom Prinzip der Einstaatlichkeit plädiert. ${ }^{33}$ Diese weite Fassung wurde bei den nicht derart als mißbrauchsanfällig gesehenen gesetzlichen Verlustgründen nicht fortgesetzt: Der fortdauernde Wille zur Zugehörigkeit zum deutschen Staatsverband solle bei Eingliederung in eine andere Staatszugehörigkeit unbeachtlich sein können; nur drohende Staatenlosigkeit hindert den gesetzlichen Verlust gegen den Willen der Einzelnen. ${ }^{34}$

\subsection{Auslieferung und Asyl'3}

Das Verbot der Auslieferung Deutscher - hier also einschließlich der Statusdeutschen - an das Ausland knüpft an Art. 112 WRV an - mit einer bemerkenswerten Variante: Mit der Abkehr vom Verbot der Überantwortung an eine ausländische Regierung sollten Auslieferungen in die SBZ und an die (westlichen) Besatzungsmächte grundgesetzlich möglich bleiben. ${ }^{36}$ Die Möglichkeit einer Auslieferung an die SBZ und damit die » Tragik unserer staatsrechtlichen Situation $\langle\ldots\rangle$, daß wir kein Deutschland haben ${ }^{37}$, brachte den Allgemeinen Redaktionsausschu $\beta^{38}$ denn auch dazu, das zuerst vom Grundsatzausschuß - in der von Carlo Schmid gefundenen Formulierung vorgeschlagene - weitgefaßte Asylrecht für politisch Verfolgte ${ }^{39}$ bei Ausländern wieder auf ein Auslieferungsverbot zu beschränken ${ }^{4}$; das Asylrecht sollte im Bundesgebiet Deutschen vorbehalten werden, die wegen ihres »Eintretens

32 Hier hat der Grundsatzausschuß bewußt an Art. 3 E-Menschenrechtskonvention angeknupft (Grundsatzausschuß 26. Sitzung v. 30.11.1948, KurzProt 1; s.a. Hauptausschuß, 18. Sitz. v. 4.12.1948, Prot. 216 f.; der Entwurf lag dem PR als Abdruck aus der "Neuen Zeitung v. 7. 10.1948 (PR 10.48I 44/III > vor), wobet zunachst insb. der Entzug wegen "Verrats am Vaterland« (politische Gründe, z. B. Eintritt in eine fremde Wehrmacht oder eınen auslandischen Geheımdienst) thematisiert wurde, ferner das Prinzip der Vermeidung von Staatenlosigkeit (PR $12.4^{8}-33^{8}$ II). Klar ist jedenfalls, daß bloßen Statusdeutschen der Ausburgerungsschutz nicht zukommen kann.

33 Abg. Schmid, Hauptausschuß-Steno $58 \mathrm{r}$.

34 Die Differenz von Staatsangehorigen und Statusdeutschen bewirkt, daß der Bestandsschutz des Art. 16 Abs. I S. I GG nur fur deutsche Staatsangehorige greifen kann.

35 Vgl. Hans Kreuzberg/Volker Wahrendorf, Grundrecht auf Asyl. Materialien zur Entstehungsgeschichte, 2. Aufl., Koln u. a. 1992.

36 Dies kritisierte bis zuletzt vor allem der Abg. Seebohm, der eine Überantwortung an die Besatzungsmachte aufgrund des Besatzungsstatuts als einen vom GG nicht auch noch anzuerkennenden, »von der Gegenseite zu verantwortenden Eingriff in die menschlıchen Grundrechte der Deutschen " (Hauptausschuß-Steno s82) sah.

37 Abg. v. Brentano, Allgemeiner Redaktionsausschuß, StenProt. 583.

$3_{8}$ Drs. $11.4^{8}-282$ /II, S. 3 (Allgemeiner Redaktionsausschuß, I6. I1. 1948); die Formulierung des Grundsatzausschusses erschien auch deswegen "als zu weitgehend ", weil »sie moglicherweise die Verpflichtung zur Aufnahme, Versorgung usw. in sich schließt«; in leicht geanderter Fassung auch Beschl. v. I 3. 12. 1948 , PR-Drucks. $12.48-370$, S. I.

39 Grundsatzausschuß, 4. Sitz. v. 23. 12. 1948, Sten.Prot. S. 39

40 Hier stand auch die Sorge des Allgemeinen Redaktionsausschuß Pate, die Gewahrung des Asylrechts fur Auslander ses wegen möglicher Pflichten zur Aufnahme, Versorgung usw. zu weitreichend (PR Drs. I I. $48-282$ II). 
für Freiheit, Demokratie, soziale Gerechtigkeit oder den Weltfrieden verfolgt werden $^{41}$ (Gesinnungsasyl; subjektive Lösung). Die subjektive Lösung hat der Abg. Brentano vor allem in der zentralen Asylrechtsdebatte im Hauptausschuß $\beta^{42}$ verfochten in der Sorge, Deutschland könne zur »Oase auch derjenigen politisch Verfolgten werden, die ihre Tätigkeit, die sie zum Abwandern aus der Heimat veranlaßt hat, auch hier fortsetzen werden, nämlich den Kampf gegen die Demokratie«.

Diese enge Fassung hat sich nicht durchgesetzt. Das hergebrachte Auslieferungsverbot wurde vielmehr unter Transformation des völkerrechtlichen Asylrechts ${ }^{43}$ in das bewußt als Grundrecht ausgestaltete Asylrecht für alle politisch Verfolgten fortentwickelt. Der klassisch nationalstaatliche Rahmen wurde hierdurch nicht völlig aufgegeben. Denn von dem Sonderproblem der in der SBZ politisch Verfolgten abgesehen, bestand Einigkeit: Die Zufluchtsuchenden können keine Angehörigen der schutzgewährenden Nation sein. ${ }^{44}$ Die Asylgewährung gliederte sie auch nicht als Aktivbürger in den Staatsverband ein, sicherte ihnen indes einen Status als Passivbürger. Die Ausformung als »Individualgrundrecht des Fremden « ging aber über den völkerrechtlichen Rahmen weit hinaus und sprengte durch das Zufluchtsuchenden einzuräumende Zugangsrecht den durch nationale Grenzen gezogenen Kordon.

Kontroverser als die Einführung des Asylrechts dem Grunde nach und die möglichen Belastungsgrenzen ${ }^{45}$ war die Diskussion um die einschränkende Qualifizierung der möglichen Verfolgungsgründe, etwa wegen des Eintretens für bestimmte Anliegen oder einer den Grundsätzen der Verfassung widerstreitenden Verfolgung. ${ }^{46}$ Die Entscheidung für das objektive Merkmal der politischen Verfolgung als Schutzvoraussetzung, ein Ordnungsasyl ${ }^{47}$ also, unter Verzicht auf jede subjektiv-gesinnungsorientierte Einschränkung, zielte bewußt auf eine generöse Asylrechtsgewährung, die die Möglichkeit des Irrtums in der Person und die Asylgewährung für (politisch) unerwünschte Ausländer ${ }^{48}$ einschließt; es war auch eine Absage an eine Prüfung durch die Grenzorgane. ${ }^{49}$ Hier hat sich letztlich in einem parteiübergreifenden Konsens der Wille durchgesetzt, nach den eigenen Erfahrungen mit politischer Verfolgung politischen Flüchtlingen - unabhängig vom Herkunftsland oder der konkreten politischen Gesinnung - wenigstens in Deutschland einen gesicherten Status zu verschaffen.

Die genaue Ausformung des Rechtsstatus der Flüchtlinge, insb. ihr Recht auf politische Aktivität und Mitgestaltung im Aufnahmestaat ist indes ebenso wie die soziale Absicherung ${ }^{\circ}$ nur am Rande angesprochen worden, etwa in dem Hinweis des Abg.

41 Art. 17 1.d. F. des Allgemeinen Redaktionsausschusses vom I3. I 2. 1948 (Hauptausschuß-Steno 2 I 8); s. a. die Wurdigung des Grundrechtskatalogs durch Thoma, PR-Drucks. $11.48-244 /$ III, S. sf.

42 44. Sitzung, 19. 1. 1949, S. 583

43 Abg. Zinn: „Der Begriff des Asylrechts ist fest umrissen durch das allgemeine Völkerrecht “ (Grundsatzausschuß, 4. Sitz. vom 23.9. 1948 , StenProt. S. 36.

44 Abg. Wagner im Allgemeinen Redaktionsausschuß.

45 Etwa Abg. Fecht (CDU): Aufnahme in unbegrenzter Zahl (Hauptausschuß I8. Sitzung vom 4. I2. $194^{8)}$.

46 So die Formulierung des Art. 4 Abs. 2 Herrenchiemseer Entwurf in Anlehnung an Art. 105 LV BY, Art. 7 LV HE und Art. 16 Abs. 2 LV RP; s. a. Art. ${ }_{4} 4$ Grundrechtskatalog Bergstrasser, der auf „Fremde« abstellt; Allgemeiner Redaktionsausschuß, Fassung vom I6. I1. 1948; Abg. Renner, PR Drs. 294 II.

47 Vgl. H.-P.Schneider, Das Asylrecht zwischen Generositat und Xenophobie, in: W. Benz (Hrsg.), Jahrbuch für Antisemitismusforschung 1, 1992, 217 (221).

$4^{8}$ Die war eınes der Motıve des Allgemeinen Redaktionsausschusses fur seine einschrankende Fassungen, PR-Drs. $12.48-370$

49 Insb. Abg. v. Mangoldt, Grundsatzausschuß. 23 . Sitzung v. 19. 11.1948 , StenProt. S. 14.

50 Der Abg. Schmıd bezeichnte dabei das Verlangen einer Militarregierung nach Unterhaltsgewahrung fur Fluchtlinge als "groteske Auslegung". Der Abg. Renner zog seinen Antrag, das Asylrecht auf das Recht auf Arbeit zu erstrecken, nach dem Hinweis auf die Geltung der allgemeinen Handlungsfreiheit zuruck 
Schmid, Asylrecht bedeute nicht Freizügigkeit ${ }^{51}$, sondern sei häufig mit Stellung unter Polizeiaufsicht verbunden..$^{52}$ Asylgewährung bedeutet mithin nach dem Willen der Verfassunggeber nicht die Teilhabe an den Rechten und Chancen, die exklusiv an die Staatsangehörigkeit gebunden sind; als geschützt yorausgesetzt wurde allein das Recht, sich durch frei gewählte Arbeit den Lebensunterhalt selbst verdienen zu können.

\section{Der sozio-ökonomische Status der Fremden}

Werden Bürger und Fremde im Staatsbürgerstatus getrennt, stellt sich die Frage nach der Exklusivität der damit verbundenen Rechte. ${ }^{33}$ Der Herrenchiemseer Entwurf war hier noch universalistischer als der Parlamentarische Rat: Bis auf das Wahlrecht waren alle Grundrechte - einschließlich der Versammlungs-, Vereinigungs- und der Berufsfreiheit im Bundesgebiet - als Menschenrechte gestaltet; lediglich das aktive und passive Wahlrecht war deutschen Staatsangehörigen vorbehalten. Eine Sonderstellung nahm schon in Herrenchiemsee das Freizügigkeitsrecht ein, auf dessen Einführung verzichtet wurde, "weil die gegenwärtigen Zustände der Durchführung unüberwindbare Hindernisse bereiten .54

Die enge Verknüpfung von Freizügigkeit und Arbeits- und Berufsfreiheit zu Beginn der Beratungen des Parlamentarischen Rats war der Schlüssel zu einer Begrenzung auch der in einer Marktgesellschaft zentralen Chancen des Zugangs zum Arbeitsmarkt auf die Deutschen. Wegen der Flüchtlingszahlen und der Situation auf dem Wohnungsmarkt sollte das der Arbeitsaufnahme vorangehende Recht, Aufenthalt und Wohnsitz zu nehmen, zunächst auf die Bundesangehörigen beschränkt werden's, um der unerfüllbaren Pflicht zu entgehen, den » I6 Millionen Deutschen östlich unserer Grenzen « ${ }^{56}$ das Freizügigkeitsrecht zubilligen zu müssen.

Hier plädierte vor allem die SPD - auch in Ansehung der verwaltungspraktischen Schwierigkeiten - aus politischen Gründen erfolgreich für eine Erstreckung auf alle Deutschen, um eine verfassungsfeste Zäsur zwischen Deutschen in Ost und West zu vermeiden ${ }^{57}$; die Sondersituation wurde durch die Einschränkungsmöglichkeiten und die Übergangsregelung in Art. 117 Abs. 2 GG berücksichtigt.

Nach der systematischen Ablösung der Arbeits- und Berufsfreiheit von der Freizü-

(Hauptausschuß ${ }_{44}$.S. vom 19. I. 1949〉, Hauptausschuß-Steno, 582, 584); der Abg. Wagner sah es als Aufgabe des Bundesgesetzgebers, den Asylberechtigten auch die Moglıchkeiten zum Leben zu schaffen. An umfassende Versorgungsanspruche war schon wegen der allgemeinen Notsituation der NachkriegszeIt nicht zu denken.

5I S. auch Abg. Eberhard, Hauptausschuß 44. Sitzung vom 19. I. 1949 (K 55).

52 Hauptausschuß, 18. Sitzung vom 4. 12. 1948.

53 Vgl. Ulrich K. Preuß, Staatsbürgerschaft und Zivilgesellschaft, KJ $1993,232 \mathrm{f}$.

54 Die Idee, auch das Auswanderungsrecht zu regeln, und zwar mit dem Ziel seiner Beschrankung, um ein "Ausbluten « des geschwachten deutschen Volkes bzw. den Verlust fur den Wiederaufbau dringend benotigter Personen zu verhindern, wurde als politssch zu brisant fruhzeitig fallengelassen (Grundsatzausschuß, 5. Sitzung vom 29.9. 1948, Nr. 8/103)

55 Grundsatzausschuß, Entwurf vom 19. 11 . 1948, Drs. 294: "Alle Bundesangehorigen genießen Freizugigkeit im ganzen Bundesgebiet. Sie haben das Recht, an jedem Ort des Bundesgebietes Aufenthalt und Wohnsitz zu nehmen. «; so auch Fassung vom I, 12.1948 (2. Lesung Grundsatzausschuß; Anlage zum KurzProt der 27. Sitzung vom 1. 12. 1948 〈PR 1, $48-326$ II $\rangle$ ); Hauptausschuß I 8. Sitz. v. 4. 12.1948 , KurzProt 2 (PR I.49-456〉).

56 Von Mangoldt, Grundsatzausschuß, StenProt. S. Is.

57 Abg. Schmid, Hauptausschuß, 44. Sitzung v, 19. 1. 1949, StenProt. S. 573 f. (KurzProt 4): Es gehe nicht allein um administrative, sondern auch um politısche Gesichtspunkte; man solle eine unnotige Zasur zwischen Ost und West vermeiden und daran denken, daß man eıne Organisation des ganzen deutschen Volkes schaffen wolle. Dr. Seebohm verwies auf die zahlreichen Volksdeutschen, die sich in den ost I $_{1}$ hen Landern aufhielten und denen man das Recht gewahren musse, im Bundesgebiet Aufenthalt und Wohnsitz zu nehmen; der Hauptausschuß beschloß mit großer Mehrheit sodann, die Freizugigkeit den Deutschen zuzubilligen (19:0:2). 
gigkeit verblieb es ohne intensivere Erörterungen bei der Beschränkung dieser ökonomischen Freiheit auf Deutsche bzw. Bundesbürger. ${ }^{8}{ }^{8}$ Universeller Schutz wurde lediglich vor $Z$ wangsarbeit geboten.

Bewußter war die Beschränkung der allgemeinen Vereinigungsfreiheit auf die Deutschen, die sich schon früh im Grundsatzausschuß durchsetzte ${ }^{59}$. Vorbild war Art. I 24 WRV; auf vergleichbare Beschränkungen auf Landesangehörige im Ausland (Belgien) wurde verwiesen. Gründe für die Beibehaltung der Koalitionsfreiheit als Menschenrecht sind den Debatten indes ebensowenig zu entnehmen wie für die vom Grundsatzausschuß beschlossene Reduktion der Versammlungsfreiheit auf Deutsche. Ausländern sollte damit nicht jede Versammlungsfreiheit, sondern nur deren Verfassungsschutz genommen werden. ${ }^{60}$

Bei der Gleichheit vor dem Gesetz hatte der Allgemeine Redaktionsausschuß ohne nähere Begründung ${ }^{61}$ eine auf Deutsche beschränkte Fassung vorgeschlagen. ${ }^{62}$ Der Grundsatzausschuß blieb hier seiner universelleren Grundhaltung treu und erhob den allgemeinen Gleichheitssatz zum Menschenrecht. ${ }^{63}$ Das besondere Diskriminierungsverbot war ebenfalls mit seiner Schutzwirkung nicht auf Deutsche begrenzt. ${ }^{64}$

\section{Staatsbürgerliche Gleichbeit}

Die Beschränkung der politischen Teilhabe auf die Deutschen verdeutlicht Art. 33 GG, der die staatsbürgerliche Gleichheit in Rechten und Pflichten über die Ländergrenzen hinaus auf die Deutschen begrenzt und dies mit der Beschränkung des verfassungsgesetzlich gesicherten Zuganges auf den öffentlichen Dienst verbindet. Der Herrenchiemseer Entwurf hatte mit der Begrenzung des Wahlrechts auf die Staatsbürger nur einen Teilaspekt herausgegriffen und im übrigen den öffentlichen Dienst dem Wortlaut ${ }^{65}$ nach auch Nichttdeutschen geöffnet.

Der Grundsatzausschuß gab dieses Konzept ohne nachvol]ziehbaren Grund auf. Er knüpfte schon früh ${ }^{66}$ mit einer zunächst auf Deutsche ${ }^{67}$ und dann zeitweilig auf Bundesangebörige ${ }^{68}$ abgestellten Formulierung ${ }^{69}$ zur staatsbürgerlichen Gleichheit

58 Nach der Ausdehnung der Freizugıgkeit auf alle Deutschen wurde die Erstreckung auf die Berufsfreiheit als redaktionelles Folgeproblem behandelt (Abg. de Chaphaurouge, Hauptausschuß 44. Sitz. y. 19. 1. 1949, KurzProt 5, Prot.575)

59 6. Sitzung v. 5. 10. 1948 , KurzProt zu Drs. 148, S. 2 f.

60 Abg. Zinn, Grundsatzausschuß, 6. Sitzung v. 5. 10. 1948, KurzProt zu Drs. 148, 9 f.

61 Für den Wechsel wird ledıglich auf die in den Ubergangsbestimmungen gegebene Begriffsbestımmung verwiesen. Protokolle des Allgemeinen Redaktionsausschussés hierzu sind nicht greifbar.

62 Art. 1b in der Fassung des Algemeinen Redaktionsausschusses vom I6.1 1. 1948, PR I1.48-282 II.

63 26. Sitzung vom 30 . 1 I. 1948 , KurzProt 3 ( $P_{12}$ 12.4 - 338 II).

64 So bereits in der vom Grundsatzausschuß am 1. 12. $194^{8}$ beschlossenen Fassung, die mit der Endfassung ubereinstimmt.

6) Art. 13 Herrenchiemseer Entwurf: Die offentlichen Ämter stehen jedem nach Maßgabe seiner Befahigung und Eignung offen.

66 6. Sitzung v. 5. 10. 1948.

67 Kontext war u. a. auch die Frage einer Vermittlung der deutschen Staatsangehorigkeit durch eine Landerstaatsangehorigkeit (s. etwa Grundsatzausschuß VIII/7.10.1948, KurzProt. 3 f.); die Offnung für Nichtdeutsche stand nicht ernsthaft zur Debatte. Mit der Diskussion um die Länderstaatsangehórigkeit verbunden war die Frage nach der Gesetzgebungszustandigkeit fur die Staatsangehorigkeit in Bund und Landern; die landergrenzenubergreifende Gleichheit aller Deutschen in Rechten und Pflichten sowie beim Amterzugang betonen die Textfassungen des Grundsatzausschusses (Art. 27a Fassung vom 10. 12. 1948, PR 12.48 - 340) und des Allgemeinen Redaktionsausschusses (Art. 19, 27 a der Stellungnahme zum Hauptausschuß, Drucks. Nr. 370 ).

68 Fassung 18. 10. 1948 (PR. 10.48- PR. 203). Der Sache nach ging der Streit um die Einbeziehung bzw. den Ausschluß der in Ostdeutschland lebenden Deutschen; s. nur Abg. v. Mangoldt, 27. Sitzung des Hauptausschusses v. I 5. I 2. 1948, einerseits, Abg. Seebohm andererseits (KurzProt S. 12; Prot. S. 326); ahnlich auch der Abg. Renner. Der Hauptausschuß bleibt dann gegen den Grundsatzausschuß bei der Fassung, die alle »Deutschen« begünstigte.

69 Art. 13. 
an die Weimarer Reichsverfassung an ${ }^{70}$. Die Binnenperspektive betonte die föderale Dimension dieses Gleichheitsgebotes: In der 6. Sitzung bewegte den Grundsatzausschuß die Frage sicherzustellen, daß Nichtangehörige eines Bundeslandes in den Verwaltungsdienst eines anderen Bundeslandes eingestellt werden können ${ }^{71}$. Die Bezugsgröße der "gleichen staatsbürgerlichen Rechte«, nämlich »die Angehörigen des Landes «, ist erst auf Vorschlag des Fünferausschusses entfallen und so vom Hauptausschuß in der 4 . Lesung beschlossen..$^{72}$ Bei den Deutschen-Grundrechten setzte sich die Ausdehnung der Grundrechtsträgerschaft über die Bundesangehörigen hinaus auf alle Deutschen ${ }^{73}$ letztlich durch. Den Zusammenhang zwischen Staatsbürgerschaft ${ }^{74}$ und Steuerpflicht, mit dem der Allgemeine Redaktionsausschuß $3^{75}$ erfolgreich den Vorschlag zur Streichung der Beschränkung der Rechte und Pflichten auf nur ein Bundesland begründete, wurde auf die Nichtdeutschen nicht übertragen: An ihrer Steuerpflichtigkeit kam kein Zweifel auf.

Bei der exklusiven Garantie gleichen Zugangs zum öffentlichen Dienst nur für Deutsche standen die politische Neutralität des öffentlichen Dienstes und das Verbot der Diskriminierung aus Gesinnungsgründen im Vordergrund. Auch die Interventionen der Alliierten Militärgouverneure ${ }^{7}$ verlangten gleichen Zugang zum unpolitischen öffentlichen Dienst nur für »jeden Bürger «"7, ohne den Staatsbürgerbegriff hier oder sonst zu klären.

Einen speziellen Bezug zur deutschen Staatlichkeit erhalten diese Regelungen durch die Verbindung mit der verfassungsgesetzlichen Gewährleistung des Berufsbeamtentums, dem Funktionsvorbehalt und der Garantie der hergebrachten Grundsätze des Berufsbeamtentums, durch den Fünferausschu $\AA^{7}{ }^{8}$. Objektivität und Neutralität des Berufsbeamtentums wurden bewußt gegen die »Verhältnisse im Osten « ${ }^{79}$ gewendet und dafür auch die Anknüpfung an durchaus nicht nur positiv gewertete deutsche Traditionen eines Beamtentums mit besonderen Dienst- und Treuebindungen akzeptiert und gegen die Alliierten durchgesetzt. Die Vorstellung, deutsche Hoheitsgewalt könnte durch beamtete Ausländer ausgeübt werden, lag dem Parlamentarischen Rat dabei fern.

\section{Schlußbemerkung}

Unter dem Einfluß des Entwurfs der Allgemeinen Erklärung der Menschenrechte zeigt sich im Grundgesetz ein der Weimarer Reichsverfassung unbekannter universalistischer Grundzug, was den Schutz Nichtstaatsangehöriger betrifft. Menschenwürde und Gleichheitssatz sind Menschenrechte, das Asylrecht als spezielles »Fremdengrundrecht« öffnet ein Stück weit den nationalstaatlichen Panzer. Die po-

70 Art. 109 WRV verbindet den auf Deutsche beschrankten Gleichheitssatz mit dem geschlechtsbezogenen Gebot grundsatzlich gleicher staatsburgerlıcher Rechte und Pflichten.

71 KurzProt zu Drs. 148/II v. 5. 10. 1948, 3.

72 Vgl. Drs. 3.49-675 〈Gegenuberstellung Hauptausschuß - 3. Lesung - Stand 10. 2. 1949 - und Funferausschuß Stand 28. 2. 1949; Hauptausschuß, 4. Lesung (5.5. 1949), Kurzprot. Drs. 932; Sten. Prot. 751. 73 Hauptausschuß, 27. Sitzung vom 15. 12.1948.

74 Das Grundgesetz kennt - anders als etwa Art. 7 LV BY, Art. 75 LV RP - die von der Staatsangehorigkei unterschiedene Staatsburgerschaft nicht als besonderen Verfassungsbegriff; wegen der Burgerschaftsrechte, die den nicht zum Organvolk zahlenden "Statusdeutschen " zustehen, 1st die Staatsangehórigkeit nur mit Einschrankung »selbstverstandliche Voraussetzung der Staatsburgerschaft « (so Grawert $\langle$ Fn. 4 ) Rdn. ${ }^{8} 8$ ).

75 Aligemeiner Redaktionsausschuß, 25. 1. 1949, Drs. PR $1.49-543$ (Stellungnahme zu der in 2. Lesung vom Hauptausschuß geschlossenen Fassung des GG-Entwurfes〉.

76 Memorandum vom 22.11. 1948, lit. g) und h); Memorandum vom 2.3. 1949, Nr. 8.

77 Engl.: each citizen; franz.: tout citoyen.

78 Vorschlag vom 5. 2.1949, Drs. 591

79 Abg. Strauß, I2. Sitzung Zust A vom 14. 12.1948, Drs. 75 1. 
litischen und ökonomischen Betätigungsrechte bleiben - mit Ausnahme der Meinungs- und Pressefreiheit - indes exklusiv den Deutschen vorbehalten und unterstreichen so die Bedeutung, die der Art. 116 GG für den Zugang zum Staatsbürgerverband hat. Der Parlamentarische Rat verbleibt im Bezugsrahmen einer klassischen europäischen Nationalstaatlichkeit. Die in dieser Tradition mit angelegten universalistischen Momente werden aber durchaus betont.

Die von den Müttern und Vätern des Grundgesetzes gezogenen Lehren können in dieser Hinwendung zu universalistischen Prinzipien auch als bewußte Selbstrelativierung des Konzepts der Nationalstaatlichkeit verstanden werden. Die durch Arbeitskräfteimport, den fortschreitenden Prozeß der europäischen Integration, ${ }^{80}$ den Wegfall des "Eisernen Vorhangs" sowie durch Krieg und Armut veranlaßte Wanderungs- und Fluchtbewegungen bewirkten Spannungen zwischen geringeren politischen und ökonomischen Rechten und funktional gleichen Pflichten der auf Dauer im Lande lebenden Personen ohne deutsche Staatsangehörigkeit, verschärft durch die - nicht verfassungsfeste - Anbindung des Erwerbs der deutschen Staatsangehörigkeit an das jus sanguinis, ist vom Parlamentarischen Rat in ihrer heutigen Bedeutung nicht vorausgesehen worden. Gegenüber der relativen Offenheit, die der Parlamentarische Rat in einer historisch viel schwierigeren Zeit bewiesen hat, bedeutet die heutige Rückkehr zur Hypostasierung von Nationalstaatlichkeit einen Rückschritt. Die Bewahrung der Ansätze des Parlamentarischen Rates für die heutige Zeit verlangte vielmehr nach einer Verfassungsreform, welche insbesondere die Kluft von ökonomischen und politischen Rechten durch weitere Entkoppelung des Aktivbürgerstatus von der Staatsangehörigkeit mindert und die Entwicklung der Deutschenzu Menschenrechten vorantreibt. Die Widerstände selbst gegen ein Kommunalwahlrecht für Nicht-EG-Ausländer und der sich auf eine Achtensklausel beschränkende Vorschlag der Gemeinsamen Verfassungskommission von Bundestag und Bundesrat für einen Minderheitenartikel ${ }^{81}$ zeigt, wie wenig die Politik heute von der auch zukunftsweisenden Kraft einer Verfassung, wie sie den Müttern und Vätern des Grundgesetzes vorgeschwebt hat, verstanden hat.

\section{Joachim Perels \\ Ein Fremder im eigenen Land: \\ Robert M. W. Kempner (1899-1993)}

Robert M. W. Kempner war bis in die Gegenwart ein Fremder in Deutschland. Nachdem er, Demokrat und Jude, als Beamter im Preußischen Innenministerium den Aufstieg der Nazis nicht verhindern konnte - er hatte 1930 wegen ihrer vielen Rechtsverletzungen in einer Denkschrift ein Verbot der NSDAP gefordert-, wurde er 1933 auf Befehl von Hermann Göring aus dem Dienst entlassen und ausgebürgert.

80 Die mit der Europäischen Union eingeführte Unionsburgerschaft geht als staatsangehörgkeitsahnliches, umfassendes Rechts- und Pflichtenverhaltnıs qualitativ uber die bisherige Trennung von (europaischer) Marktbürgerschaft und (nationaler) Staatsburgerschaft hinaus; zu Fragen eines europaischen "Volkes" s. W. von Simson, Was heißt in einer europaischen Verfassung "Das Volk"?, EuR 1991, Iff.

8I Die GVK hat die Einfügung eines neuen Art. $20 \mathrm{~b}$ (Recht ethnischer Minderheiten) mit folgendem Wortlaut vorgeschlagen: „Der Staat achtet die Identitat der ethnischen, kulturellen und sprachlıchen Minderheiten (BT-Drs. I 2/6000); s. dazu auch die weitergehenden Vorstellungen im Gesetzentwurf der SPD BT-Drucks. 12/6323; zur volker- und staatsrechtlichen Lage des Minderheitenschutzes in Europa vgl. Rainer Hofmann, Minderheitenschutz in Europa, ZaoRV Bd. 52 (1992), Iff. 